\title{
The Cytoplasmic Domain of Voltage-dependent Potassium Channels of the Eag Family May Play a Role in the Regulation of Ion Transport
}

Julia Kacher ${ }^{1}$, Grigory Gluhov ${ }^{2}$, Denis Abramochkin ${ }^{1}$ and Olga Sokolova ${ }^{3}$

${ }^{1}$ Moscow State University named after M.V.Lomonosov, Moscow, Moskva, Russia, ${ }^{2}$ Moscow State University named after M.V.Lomonosov, Sochi, Moskva, Russia, ${ }^{3}$ Lomonosov Moscow State University, Faculty of Biology, Moscow, Moskva, Russia

The potassium channel Kv10.2 belongs to the family of voltage-dependent channels ether-à-go-go $(\mathrm{KCNH})$ and is one of the least studied members of the family. Until now, the channel gating mechanism remains unclear. It is believed that they are regulated by the movement of the transmembrane voltage sensor, which is connected through the S4-S5 linker to the pore domain. These gaps in the picture of the arrangement and functioning of the Kv10 channels can be explained by a lack of experimental data. Now these proteins are actively studied using electron and cryo-electron microscopy, in particular, threedimensional structures of voltage-dependent EAG channels have already been obtained [1]. In EAG channels, unlike the Shaker family, there is no Pro-X-Pro motif in S6, and the S4-S5 linker is a short loop consisting of five residues. Its functioning as a mechanical lever is unlikely. EAG channels have especially long cytoplasmic $\mathrm{N}$ - and C-terminal domains, which interact with each other and some cytoplasmic proteins which affect the functioning of the channels. The C-terminus performs several functions: it is responsible for the expression, folding, channel permeability, as well as for the folding direction and specificity of binding with partners; it contains a homolog of the nucleotide binding domain (cNBHD) and a tetramerization CAD domain. It has recently been established that Kv10.2 channels with a deleted $\mathrm{N}$-terminus remain capable of clustering with actin, which suggests that the $\mathrm{C}$-terminus is probably involved in the interaction with actin [2]. It was also found that the C-terminal domain can interact with $\alpha$ - and $\beta$-tubulin. The structure of the C-terminus of the EAG2 channel (723-988 aa) has not yet been solved with high resolution.

We obtained a mutant hEag2 channel with a truncated region at the C-terminus of 129 mobile amino acids $(\Delta 742-870)$. This part of the C-terminus is a mobile cytoplasmic loop with no reported functions. The channel protein was successfully expressed in eukaryotic cells, which was confirmed by Western blot analysis (Figure 1). Next, we performed electrophysiological studies using the patch-clamp method. We also conducted a study of the distribution of the channel inside the $\mathrm{CHO}$ cell and there were no abnormalities in it (Figure 2). There were no currents in the mutant channel. This kind of truncation was already shown for rKv10.1 channel, but in the study [3] the activity of the channel remained. These facts show that there is a hidden significance beneath this unstructured region [4].
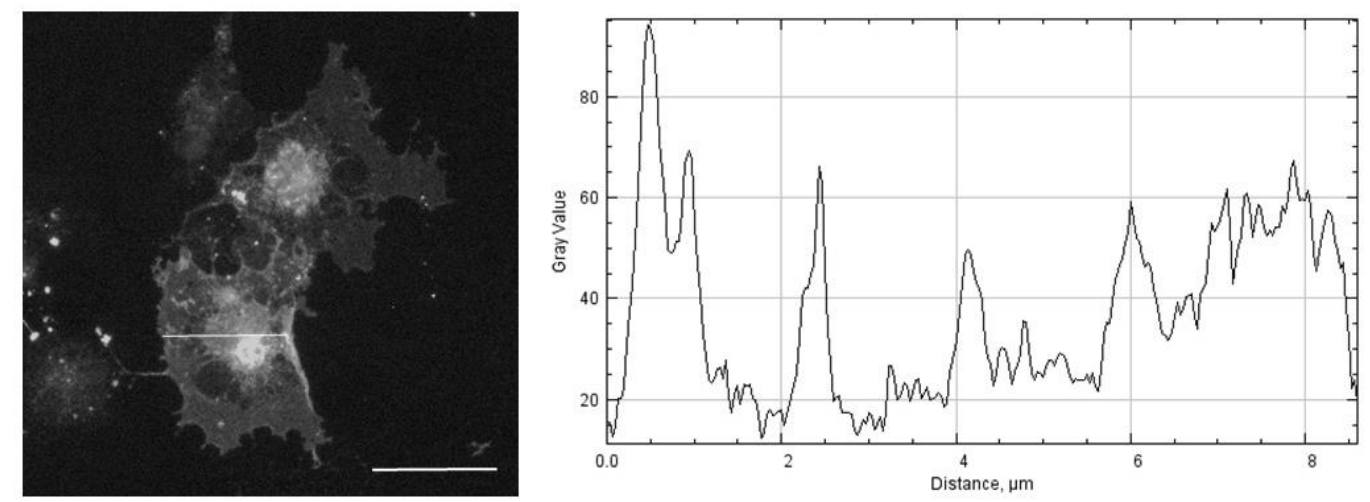
Figure 1. The expression of $\mathrm{Kv} 10.2 \Delta \mathrm{C}$ channel ion channels in eukaryotic cells. The fluorescence images of channels labeled by antibodies against the C-terminal 1D4 tag (on the left). Intensity profiles are along the white line that crosses the image (on the right). The scale bar is $10 \mu \mathrm{m}$.

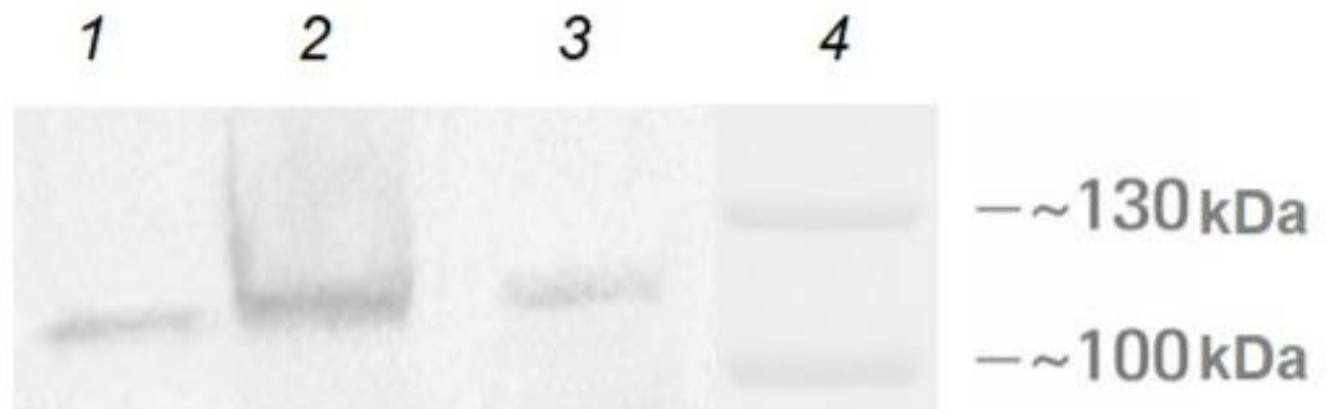

Figure 2. Immunoblot analysis of samples from transfected cos-1 cells containing a sequence of 1) fullsize Kv10.2 channel with 1D4, via electroporation 2) full-size Kv10.2 channel with 1D4 channel, via lipofection 3) trunked channel, via lipofection 4) molecular weight marker

References

[1] Wang W., MacKinnon R., 2017, Cell 169, 422-430.

[2] G. S. Glukhov, A. V. Popinako, A. V. Grizel et al., Biophysics. 2016, 61, 4, 591-595.

[3] Whicher J, MacKinnon R., Science. 2016, 12, 353(6300), 664-9.

[4] The work was supported by RFBR, project 18-74-00087. The research was carried out using the equipment purchased on account of the Lomonosov MSU Development Program. 